\title{
Ultrafiltration Evaluation \\ With Depleted Uranium Oxide
}

$$
\begin{gathered}
\text { REOENED } \\
\text { APR } 231998 \\
\text { OSTI }
\end{gathered}
$$
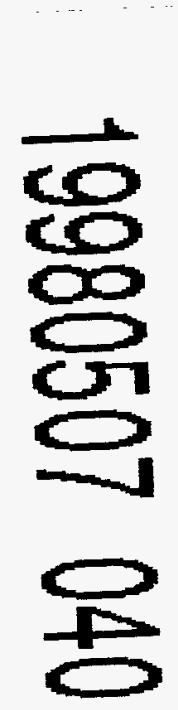

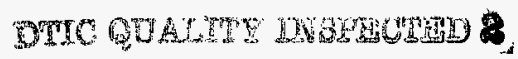

\section{Los Alamos}

NATIONAL LABORATORY

Los Alamos National Laboratory is operated by the University of California for the United States Department of Energy under contract W-7405-ENG-36. 
Edited by Margaret Burgess, Group CIC-1

An Affirmative Action/Equal Opportunity Employer

This report was prepared as an account of work sponsored by an agency of the United States Government. Neither The Regents of the University of California, the United States

Government nor any agency thereof, nor any of their employees, makes any warranty, express or implied, or assumes any legal liability or responsibility for the accuracy, completeness, or usefulness of any information, apparatus, product, or process disclosed, or represents that its use would not infringe privately owned rights. Reference herein to any specific commercial product, process, or service by trade name, trademark, manufacturer, or otherwise, does not necessarily constitute or imply its endorsement, recommendation, or favoring by The Regents of the University of California, the United States Government, or any agency thereof. The views and opinions of authors expressed herein do not necessarily state or reflect those of The Regents of the University of California, the United States Government, or any agency thereof. Los Alamos National Laboratory strongly supports academic freedom and a researcher's right to publish; as an institution, however, the Laboratory does not endorse the viewpoint of a publication or guarantee its technical correctness. 
Ultrafiltration Evaluation

With Depleted Uranium Oxide

Kirk R. Weisbrod

Ann R. Schake

Arthur N. Morgan

Geraldine M. Purdy

Horacio E. Martinez

Timothy O. Nelson

DISTRIBUTION OF THIS DOCUMENT IS URLMMTED MASTR<smiles>CCCCCC</smiles> 


\title{
ULTRAFILTRATION EVALUATION WITH DEPLETED URANIUM OXIDE
}

\author{
by \\ Kirk R. Weisbrod \\ Ann R. Schake \\ Arthur N. Morgan \\ Geraldine M. Purdy \\ Horacio E. Martinez \\ Timothy O. Nelson
}

\begin{abstract}
Scientists at the Los Alamos National Laboratory Plutonium Facility are using electrodissolution in neutral to alkaline solutions to decontaminate oralloy parts that have surface plutonium contamination. Ultrafiltration of the electrolyte stream removes precipitate so that the electrolyte stream to the decontamination fixture is precipitate free.
\end{abstract}

This report describes small-scale laboratory ultrafiltration experiments that we performed to determine conditions necessary for full-scale operation of an ultrafiltration module. Performance was similar to what we observed in the ferric hydroxide system. At 12 psi transmembrane pressure, a shear rate of $12000 \mathrm{sec}^{-1}$ was sufficient to sustain membrane permeability.

Ultrafiltration of uranium(VI) oxide appears to occur as easily as ultrafiltration of ferric hydroxide. Considering the success reported in this study, we plan to add ultrafiltration to the next decontamination system for oralloy parts.

\section{INTRODUCTION}

The United States has initiated the removal of 200 tons of fissile material from its nuclear weapons stockpile. Oak Ridge enriched uranium (oralloy) constitutes a significant portion of this material for decontamination and consolidation. Los Alamos scientists have developed an electrolytic process for removing a 1-10 $\mu \mathrm{M}$ layer of oralloy by electrodissolution. Under neutral to alkaline conditions, the contaminants are removed and tend to remain with the oralloy precipitate consisting of $\mathrm{UO}_{3} \cdot 2 \mathrm{H}_{2} \mathrm{O}$. 
Within the existing electrolytic process, electrolyte containing the precipitate is recirculated to the decontamination fixture where electrodissolution takes place. After electrodissolution is complete, a high velocity stream of wash water removes any remaining precipitate from the oralloy part.

To improve system efficiency and simplify the rinsing step, ultrafiltration has been proposed as the process to continuously clean a portion of the electrolyte so that the electrolyte stream to the decontamination fixture is precipitate free. This study was initiated to evaluate the feasibility of this approach and to define the operating conditions that will lead to sustainable operation of a full-scale ultrafiltration module.

\section{EXPERIMENTAL APPARATUS AND PROCEDURE}

Concentration of precipitate in solution is a key parameter to evaluate necessary conditions for successful ultrafiltration. This value is determined from the amount of uranium removed from each part, the number of parts processed before the precipitate is removed from solution, and the total volume of electrolyte in the flow loop.

Los Alamos scientists' experience has shown that dissolution at $30 \mathrm{~A}$ for $5 \mathrm{~min}$ is sufficient to decontaminate oralloy parts to $<20 \mathrm{dpm} \mathrm{Pu}$ swipable. In the depleted uranium (DU) electrodissolution study, we found that uranium(VI) is formed at nearly $100 \%$ current efficiency. ${ }^{1}$ Under these conditions, about $3.7 \mathrm{~g}$ of uranium would be removed per part. If we assume that five parts will be processed before the precipitate is removed, a total of $18.5 \mathrm{~g}$ of uranium as precipitate will be contained in an estimated electrolyte volume of $8 \mathrm{~L}$. This calculation gives a maximum concentration of $2.3 \mathrm{~g} \mathrm{DU} / \mathrm{L}$. The total weight of precipitate is greater because of bound oxygen and water of hydration.

In order to minimize the amount of precipitate needed in the ultrafiltration experiment, a small ultrafiltration unit was constructed for operation within the Los Alamos Plutonium Facility. This unit operated well with only $500 \mathrm{ml}$ of solution. Thus, $1.156 \mathrm{~g}$ of DU precipitate (sufficient for $500 \mathrm{ml}$ solution) was produced as the final step in a series of experiments described in another Los Alamos report. The precipitate was produced in $500 \mathrm{ml}$ of $0.4 \mathrm{M} \mathrm{NaNO}_{3}$ solution. A $0.4 \mathrm{M}$ solution of $\mathrm{NaNO}_{3}$ was chosen instead of the $200 \mathrm{~g} / \mathrm{L}$ concentration since previous experiments showed that the current efficiency remains near unity over a whole range of electrolyte concentrations. ${ }^{1}$ As long as the $0.4 \mathrm{M}$ solution does not unduly increase solution resistivity, the 
lower concentration will increase ultrafiltration rates and reduce the total amount of salt contaminating the rinse water system.

The system, shown in Figure 1 consisted of the following components.

1. 1/2 gal. multipurpose jar (Cole Parmer, pn E-06761-20)

2. $\quad 0-1.7 \mathrm{gpm}$ micropump variable speed gear pump (Ryan Herco, HGA-152-0036)

3. $\quad 0-2 \mathrm{gpm} 60 \mathrm{~mm}$ flowmeter (Cole Parmer, pn E-03248-48)

4. $\quad 10-120 \mathrm{ml} / \mathrm{min} 100 \mathrm{~mm}$ flowmeter (Cole Parmer, pn E-32461-32)

5. $\quad 40-400 \mathrm{ml} / \mathrm{min} 100 \mathrm{~mm}$ flowmeter (Cole Parmer, pn E-32461-36)

6. 0-25 psi backpressure regulator, $\mathrm{CV}=1.2$ (Precision Fitting \& Gauge Co., pn BP8-1A11L9D114)

7. $\quad 0-30$ psi pressure gauges (Cole Parmer, pn E-68022-22)

8. $3 / 8$ in. i.d. tubing for main flow loop and $1 / 4$ in. o.d. tubing for permeate loop

9. Ultrafiltration module (A/G Technology Co., UP-500-E-4A)
a) $500 \mathrm{~K}$ pore size
b) $1 \mathrm{~mm}$ i.d. fiber size
c) $\quad 0.042 \mathrm{~m}^{2}$ surface area

Two circulation loops exist in the unit. A gear pump with positive suction capability displaces precipitate-containing solution through the tube side of the ultrafiltration module. Flow rate is controlled by the variable speed pump and monitored by the variable area flowmeter. Average transmembrane pressure (TMP) is set with the backpressure regulator. In the second loop, permeate, which passes through the ultrafiltration fibers, is collected on the shell side of the module and passes through one of two flowmeters before returning to the reservoir.

Two primary variables in an operating procedure include shear rate at the wall of a fiber and the TMP. Momentum flux, $\tau_{R}$, through the wall of the tube is the force per unit area that is applied to the wall of the tube by the flowing fluid. It represents the force available to remove a precipitate from the wall. Through a momentum balance, we can readily find that

where

$$
\tau_{\mathrm{R}}=\frac{\mathrm{D}\left(\mathrm{P}_{\mathrm{o}}-\mathrm{P}_{\mathrm{L}}\right)}{4 \mathrm{~L}}
$$

$\mathrm{D}=$ tube diameter,

$P_{0}-P_{L}=$ pressure drop down the tube, and

$\mathrm{L} \quad=$ tube length. 


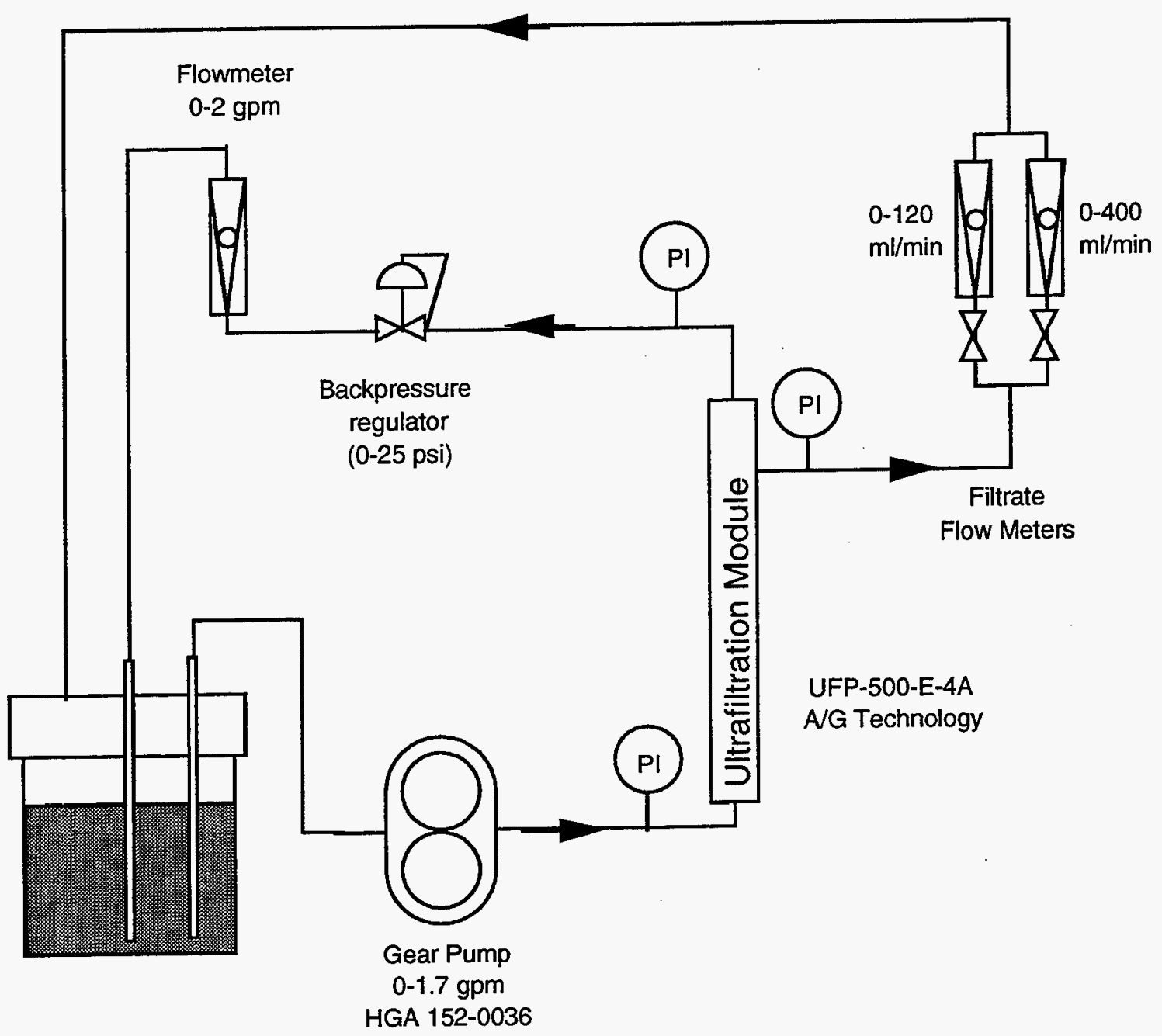

Figure 1. Flow schematic of small-scale ultrafiltration module.

Module manufacturers often normalize the flux with solution viscosity, $\mu$, to obtain the shear rate defined as

$$
\text { Shear Rate }=\frac{\tau_{\mathrm{R}}}{\mu}
$$

Guidelines are given in the A/G Technology Operating Guide for their membranes. ${ }^{2}$ The TMP is defined as

$$
T M P=\frac{\left(P_{o}-P_{L}\right)}{2}-P_{\text {permeate }}
$$

TMP represents the average driving force for depositing a precipitate on the wall. 
Equations (1) and (3) represent competing processes. A high pressure drop (high flow velocity) down the membrane tubes improves the ability of the membrane to suspend any precipitates. An increase in TMP and corresponding permeate flux tends to enhance fouling rate. A good design optimizes these two factors.

The goal of the ultrafiltration process is to obtain sufficient permeate flux with the lowest system size and weight. Because the pump represents the primary weight component, we would like to limit its size to $1 / 2 \mathrm{hp}$, if possible, for the full-sized unit.

Operating procedures for the ultrafiltration unit were based upon experience with the ferric hydroxide precipitate system that we designed to produce about $1 \mathrm{gpm}$ of permeate. ${ }^{3}$ For a TMP of $12 \mathrm{psi}$, a shear rate of $12000 \mathrm{sec}^{-1}$ was sufficient to maintain the membrane flux rate. While higher TMP yields greater flux rates, significantly higher shear rates were required to prevent fouling, thus nullifying the advantage. The $12 \mathrm{psi}$ average TMP was also convenient since it could be obtained with 20 psi or less inlet pressure, a value easily developed by a centrifugal pump. In addition, pressure drop from flow down the membrane fibers was sufficient so that a backpressure regulator was not required. Thus, these experiments were performed with an average TMP of 12 psi.

Once an initial TMP was chosen, experiments began at a high shear rate, then stepped down until permeate flow declined significantly and was not stable with time. An initial flow rate of $1.5 \mathrm{gpm}$ provided a higher shear rate than the design point for the ferric hydroxide precipitate system.

\section{RESULTS AND DISCUSSION}

Flow tests were performed over three days in an open front hood. Operating conditions and membrane performance are summarized in Figure 2. Transmembrane pressure was maintained at $12 \mathrm{psi}$. Retentant flow rate was initiated at $1 \mathrm{gpm}$ and decreased in steps to a final value of $0.5 \mathrm{gpm}$. For ultrafiltration modules of $4 \mathrm{~A}$ size, a retentant flow rate of $0.95 \mathrm{gpm}$ corresponds to the design point for the ferric hydroxide precipitate system. A permeate flux of at least $20 \mathrm{~L}-\mathrm{cp} / \mathrm{hr}-$ $\mathbf{M}^{2}$-psi is desired to obtain a total flux of $1 \mathbf{g p m}$ on the full-scale system.

After about $2 \mathrm{~h}$ cumulative operating time, the unit was shut down and the electrolyte cooled to room temperature. A second shutdown occurred after $4.7 \mathrm{~h}$. After an initial reduction in permeability from a high value, permeate flux stabilized at $31 \mathrm{~L}-\mathrm{cp} / \mathrm{hr}-\mathrm{M}^{2}$-psi. (The permeate 
flowmeter read too high at higher flow rates when the ball began to revolve in the variable diameter tube and moved upward.) Reducing the flow rate to $1.2 \mathrm{gpm}$ slightly reduced the flux. An initial drop in flux with retentant flow rate is often observed and appears to represent a reversible increase in the diffusion layer concentration of precipitate that hinders permeate flow. After shutting down for two days, a further drop in permeate flux occurred.

The retentant flow rate was finally reduced to the design point of $1.0 \mathrm{gpm}$. Sustained flux indicated no further fouling of the membrane.

Operation of two ultrafiltration modules in parallel is another option that has the potential to increase total permeate flow without increasing pump size. On this scale, module flow would decrease by one half to $0.5 \mathrm{gpm}$. At $0.5 \mathrm{gpm}$, permeate flux dropped to $22 \mathrm{~L}$-cp/hr-M $\mathrm{M}^{2}$-psi. Concurrently, the retentant stream became more transparent, indicating holdup of uranyl oxide in the ultrafiltration module. From the color change, we estimate that one half of the $1.16 \mathrm{~g}$ of uranium in the precipitate was deposited in the module.

To remove the precipitate, the retentant flow rate was then increased to its maximum value. At $1 \mathrm{gpm}$ the flux remained at $10 \%$ less than before the low flow step. After $6.3 \mathrm{~h}$ cumulative run time, flow was reversed through the module by disconnecting the fittings and flipping it end-forend. After flowing at a high rate without permeate flow, the initial flux of permeate returned to its initial value, but it was unstable and declined.

After the ultrafiltration module was fouled with uranium precipitate, it fouled more readily thereafter. A greater tendency toward fouling after initial fouling was also observed with ferric hydroxide precipitate. ${ }^{3}$ Apparently, a precipitate tends to adhere more easily to itself than the ultrafiltration membrane. The membrane supplier reported a similar observation for other hydroxide precipitates. ${ }^{4}$ 


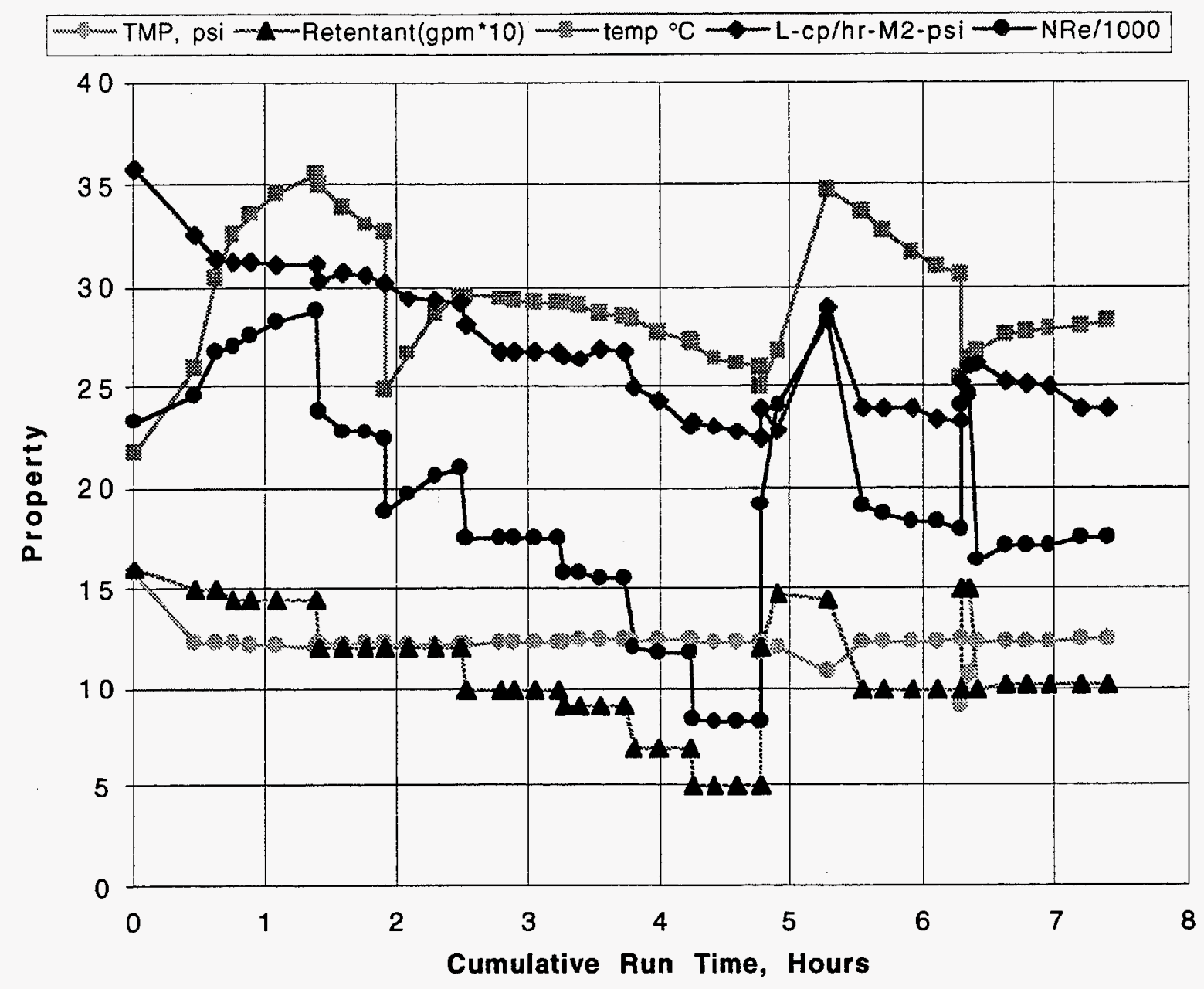

Figure 2. Performance of ultrafiltration membrane module \#4 with depleted uranyl oxide precipitate, $3.5 \mathrm{~g} \mathrm{DU} / \mathrm{L}$ of $0.4 \mathrm{M} \mathrm{NaNO}_{3} . \mathrm{N}_{\mathrm{Re}}$ represents Reynolds number.

In order to evaluate the performance of the ultrafiltration membrane, we analyzed a sample of permeate by inductively coupled plasma atomic emission spectroscopy and found it to contain $50 \mathrm{ppm}$ uranium. For comparison, thermodynamics predicts that less than $1 \mathrm{ppm}$ will be soluble at a pH of 7. ${ }^{1}$ A number of potential reasons for the disparity include:

1. While a new ultrafiltration module was used in this study, a cleaning step with $500 \mathrm{ppm}$ $\mathrm{NaOCl}$ for 2 hours at $40^{\circ} \mathrm{C}$ may have increased the mean pore size of the membrane. These conditions, however, are very near the manufacturer's recommended cleaning conditions.

2. Complex formation with $\mathrm{NaNO}_{3}$ may increase the solubility of uranium. ${ }^{5}$

3. Hydrolysis of $\mathrm{UO}_{2}^{+2}$ may increase the overall concentration of uranium species in solution. 
4. Uranium-containing colloids may be sufficiently small to pass through the ultrafiltration membrane.

While $50 \mathrm{ppm}$ uranium in the permeate does not represent a significant impediment to the process, it raises concern over the potential concentration of plutonium in solution under conditions where thermodynamics predicts that the plutonium solubility should be low.

The calculated uranium precipitate concentration of $2.3 \mathrm{~g} \mathrm{DU} / \mathrm{L}$, which was the basis of this study, was determined before new data was available from the Rocky Flats Environmental Technical Site (RFETS). At RFETS, 20-25 g uranium was removed per part to reduce contamination to an acceptable level. As a result, this study deals with a uranium concentration which is five times smaller than what might be removed from each part. Our experience with higher molar concentrations of iron may be helpful in predicting performance at higher concentrations. Stainless steel decontamination was based upon $10 \mathrm{~g} \mathrm{Fe}$ per $4.5 \mathrm{~L}$ of solution. This corresponds to a molar concentration of $0.04 \mathrm{M} / \mathrm{L}$. If we assume that iron and uranium ultrafiltration may be scaled on a molar basis, $8 \mathrm{~L}$ of $0.04 \mathrm{M} / \mathrm{L}$ corresponds to removal of $75 \mathrm{~g}$ of uranium. This is equivalent to processing a minimum of three oralloy parts. Thus, while it would be desirable to perform experiments at a higher precipitate concentration, we expect the system will perform in an acceptable manner.

\section{CONCLUSIONS}

We explored potential operating conditions for incorporation of an ultrafiltration module in the oralloy decontamination system. Performance was similar to what we observed in the ferric hydroxide system. At $12 \mathrm{psi}$ TMP, a shear rate of $12000 \mathrm{sec}^{-1}$ was sufficient to sustain membrane permeability.

Ultrafiltration of uranium(VI) oxide appears to occur as easily as ferric hydroxide. Considering the success reported in this study, we plan to add ultrafiltration to the next oralloy part decontamination system. Long-term effects of radiation upon the module are still unknown and will need to be evaluated in life tests of the system. 


\section{REFERENCES}

1. Kirk R. Weisbrod, et al., "Transpassive Electrodissolution of Depleted Uranium in Alkaline Electrolytes," Los Alamos National Laboratory report LA-13416-MS.

2. A/G Technology Corporation, Operating Guide, January 1996, p. 14.

3. K. R. Weisbrod, H. E. Martinez, and D. E. Wedman, "Evaluation of Ultrafiltration for Removal of Ferric Hydroxide Precipitates and 304 SS Dissolution Products," Los Alamos National Laboratory report LA-98-XXXX.

4. Harlan Polishook, A/G Technology Corporation, personal communication, July 1996.

5. V. M. Vdovenko, Chemistry of Uranium and Transuranium Elements, (Publishing House of the Academy of Sciences, USSR, Moscow-Leningrad, 1960), pp. 196-200. 
Report Number (14) $L A--134 / 7-M S$

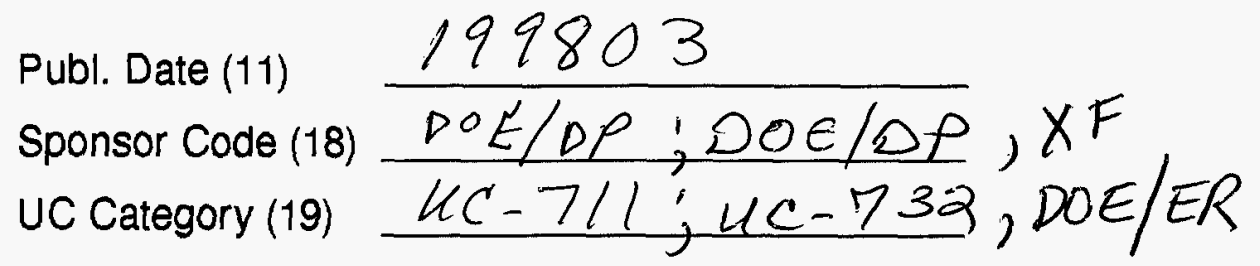

